TITLE:

\title{
Ultrasonic wave propagation in the corner section of composite laminate structure: Numerical simulations and experiments
}

\section{$\operatorname{AUTHOR}(\mathrm{S}):$}

Ito, Junta; Biwa, Shiro; Hayashi, Takahiro; Kuraishi, Akira

\section{CITATION:}

Ito, Junta ...[et al]. Ultrasonic wave propagation in the corner section of composite laminate structure: Numerical simulations and experiments. Composite Structures 2015, 123: $78-87$

ISSUE DATE:

2015-05

URL:

http://hdl.handle.net/2433/193532

\section{RIGHT:}

(c) 2014 Elsevier Ltd.; この論文は出版社版でありません。引用の際には 出版社版をご確認ご利用ください。; This is not the published version. Please cite only the published version. 
This article appeared as:

J. Ito, S. Biwa, T. Hayashi and A. Kuraishi, Ultrasonic wave propagation in the corner section of composite laminate structure: Numerical simulations and experiments, Composite Structures, Vol. 123 (2015), pp. 78-87.

\title{
Ultrasonic wave propagation in the corner section of composite laminate structure: Numerical simulations and experiments
}

\author{
Junta Ito $^{1}$, Shiro Biwa ${ }^{1 *}$, Takahiro Hayashi ${ }^{1}$ and Akira Kuraishi ${ }^{2}$ \\ 1 Department of Aeronautics and Astronautics, Graduate School of Engineering, \\ Kyoto University, Katsura, Nishikyo-ku, Kyoto 615-8540, Japan \\ 2 Aerospace Company, Kawasaki Heavy Industries, Ltd., Kakamigahara, Gifu \\ 504-8710, Japan
}

\begin{abstract}
Two-dimensional finite element simulations and experiments are presented to examine the ultrasonic wave propagation behavior in the corner section of CFRP (carbon fiber reinforced plastics) laminate structures. The numerical model consists of a right-angle corner section of CFRP laminate immersed in water and subjected to the ultrasonic wave incidence from its inner or outer side. The anisotropic stiffness constants of the unidirectional CFRP are identified experimentally and used to model the curved laminate structure. A commercial finite element analysis code is used to compute the reflection waveforms in the pulse-echo mode, and the effect of the laminate stacking sequence and the wave incidence direction is examined. It is shown that the reflection waveforms for the wave incidence from the outer side of the corner qualitatively resemble those for the plane laminate except that the echo signals of the former are substantially weaker. The numerical simulations are shown to well reproduce the qualitative features of experimental reflection measurements performed for the corner sections of 16-ply CFRP laminate. Experimental results indicate that the porosity content of the corner section can be estimated by the amplitude ratio of the surface and the back-wall echoes when a curvature-dependent calibration relation is properly used.
\end{abstract}

Keywords: Carbon fiber reinforced plastics; Curved structure; Ultrasonic wave; Finite element analysis; Porosity

* Corresponding author: Shiro Biwa, E-mail biwa@kuaero.kyoto-u.ac.jp 


\section{Introduction}

Carbon fiber reinforced plastics (CFRP) have several advantages over traditional metallic structural materials such as high stiffness-to-weight and strength-to-weight ratios. Increased applications of these composite materials, e.g. in aircraft structures, highlight the importance of nondestructive testing to ensure their structural integrity. Available techniques include the use of ultrasonic waves to detect and size different types of defects introduced during manufacturing processes. While delamination-type defects are explicitly visualized by ultrasonic echoes [1-4], distributions of minute pores, called porosity, need to be evaluated via overall changes in ultrasonic wave propagation characteristics. Foregoing studies have revealed that the porosity content of composites can be evaluated by measuring the attenuation of ultrasonic waves [5-12]. In practice, some threshold levels are preset for the height of ultrasonic echoes for each composite component to judge the porosity level on an empirical basis. For composite components with complex geometries such as corner parts or variable-thickness components, however, the feasibility of such methodologies is not a trivial issue as the wave propagation behavior becomes quite complicated.

In clarifying the ultrasonic wave propagation behavior in composite components with complex geometries and in establishing appropriate testing techniques, numerical simulations are expected to play a significant role. For example, Deydier et al. [13] used a ray-theory approach to predict ultrasonic wave propagation in a CFRP laminate of tapered thickness and found reasonable agreement with experiments. This technique was further elaborated by Journiac et al. [14] to analyze ultrasonic signals transmitted through a curved composite part. Recently, Dominguez et al. [15] and $\mathrm{Xu}$ and Zhou [16] used a finite difference technique to numerically simulate ultrasonic testing of curved composite parts by an array probe. The finite element method has also been used to analyze ultrasonic waves in anisotropic solids, e.g. [17-20]. Currently, a number of fully developed finite element codes are commercially available for numerical simulation of a variety of ultrasonic testing configurations, e.g. [21-23]. For modeling the wave propagation in curved composite laminate structures, however, one needs to account for the spatially distributed anisotropic stiffness constants over the stacking direction as well as along the curvature of the laminate structure. As a consequence there still remain a number of issues to be clarified from a fundamental point of view regarding ultrasonic wave propagation in curved composite laminate structures.

In this paper, numerical simulations and experiments are presented to elucidate the ultrasonic wave propagation behavior in the corner section of composite laminate structures. In Section 2, the computational model for a composite corner section immersed in water is described. The anisotropic elastic constants of the unidirectional CFRP necessary for the numerical modeling are identified experimentally. The two-dimensional plane-strain elastic wave propagation in the corner section is numerically simulated by a commercial finite element analysis code. Numerical results are shown in Section 3 to demonstrate the effect of the laminate stacking sequence and the wave incidence direction on the wave propagation behavior and the reflection waveform. Corresponding experimental reflection measurements are described in Section 4. Experimental results are then qualitatively compared with the numerical simulations described in Section 5. In Section 5, experimental results are also shown regarding the effect of porosity content on the reflection waveform of the corner section of composite laminates. 

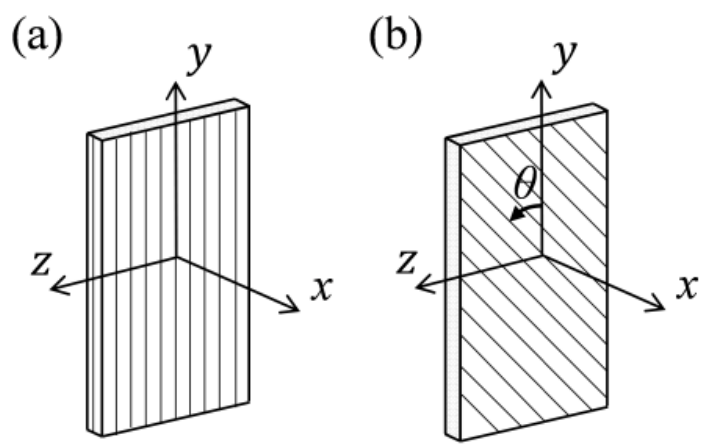

(c)

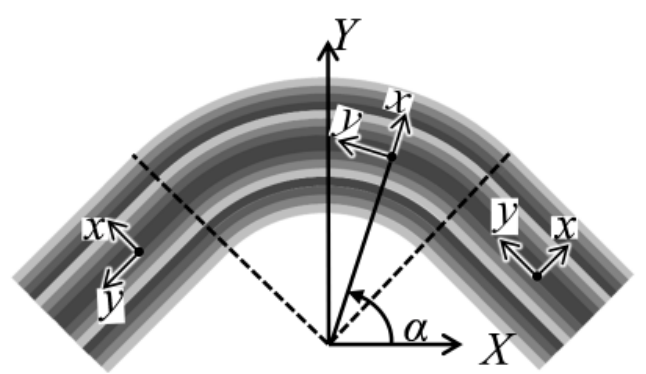

Fig. 1 A unidirectional CFRP layer, (a) when the fiber direction is along the $y$ axis and (b) when the fiber direction is rotated from the $y$ axis by angle $\theta$, and (c) a corner section of CFRP laminated structure.

\section{Numerical analysis}

2.1 Anisotropic elastic constants of unidirectional composite

In order to obtain the anisotropic elastic constants of unidirectional carbon/epoxy composite for numerical analysis, a plate specimen of 24-ply unidirectional carbon/epoxy composite laminate was fabricated using the same prepregs (Toho Tenax UTS50/135 UD) as used to manufacture the corner specimens discussed later. The thickness of the specimen was $4.53 \mathrm{~mm}$ and the other dimensions were $100 \mathrm{~mm}$ and $120 \mathrm{~mm}$. The specimen was immersed in water and subjected to an ultrasonic pulse from different directions using a non-focusing immersion probe with nominal center frequency of 2.25 MHz. Many investigators [24-29] have reported on the evaluation of anisotropic elastic constants of composites using ultrasonic waves. In this study, the double through-transmission technique [29] was used to obtain the phase velocity of the quasi-longitudinal and two quasi-transverse waves in different propagation directions. Assuming the specimen to be a homogeneous, orthotropic and linearly elastic material, its nine independent elastic constants were determined by the nonlinear least-square method which minimized the sum of the squared errors between the measured phase velocities and those predicted by Christoffel's equations, c.f. [30]. As a result, the following stress-strain relation was identified for the unidirectional CFRP, 


$$
\left(\begin{array}{l}
\sigma_{x} \\
\sigma_{y} \\
\sigma_{z} \\
\tau_{y z} \\
\tau_{z x} \\
\tau_{x y}
\end{array}\right)=\left[C^{0}\right]\left(\begin{array}{c}
\varepsilon_{x} \\
\varepsilon_{y} \\
\varepsilon_{z} \\
\gamma_{y z} \\
\gamma_{z x} \\
\gamma_{x y}
\end{array}\right), \quad\left[C^{0}\right] \equiv\left[\begin{array}{cccccc}
13.4 & 6.84 & 7.00 & 0 & 0 & 0 \\
6.84 & 120 & 7.65 & 0 & 0 & 0 \\
7.00 & 7.65 & 13.5 & 0 & 0 & 0 \\
0 & 0 & 0 & 4.88 & 0 & 0 \\
0 & 0 & 0 & 0 & 3.11 & 0 \\
0 & 0 & 0 & 0 & 0 & 4.99
\end{array}\right] \text { (in GPa). }
$$

In Eq. (1), the $x$ axis is taken along the thickness direction and the $y$ axis along the fiber direction for the convenience of the present modeling as shown in Fig. 1 (a). The mass density of the CFRP was measured as $1.54 \times 10^{3} \mathrm{~kg} / \mathrm{m}^{3}$. It is noted that viscoelastic properties of unidirectional CFRP are not accounted for in the present analysis.

The stiffness matrix $\left[C^{0}\right]$ in Eq. (1) is given with respect to the $x y z$ coordinate system when the fiber direction is along the $y$ axis. When the fiber direction is rotated with respect to the $x$ axis by an angle $\theta$ as shown in Fig. 1 (b), the stiffness components in the xyz system can be obtained by the transformation rule for a fourth-order tensor as

$$
C_{i j k l}^{x y z}=P_{i m} P_{j n} P_{k s} P_{l t} C_{m n s t}^{0},
$$

where $C_{m n s t}^{0}$ are the components of the stiffness tensor corresponding to the matrix $\left[C^{0}\right]$, and $P_{i m}$ are the direction cosines between the fiber direction and the $y$ axis. Note that the summation convention is used in Eq. (2). In the matrix representation the direction cosines are given by

$$
[P]=\left[\begin{array}{ccc}
1 & 0 & 0 \\
0 & \cos \theta & \sin \theta \\
0 & -\sin \theta & \cos \theta
\end{array}\right] .
$$

\subsection{Modeling of corner section of CFRP structure}

In accordance with the experiments described below, the CFRP laminate structure is assumed to have a right-angle corner with $3 \mathrm{~mm}$ inner radius and $3 \mathrm{~mm}$ thickness. Two types of stacking sequences are considered, namely, the 16-ply unidirectional laminate $[0]_{16}$ and the 16-ply quasi-isotropic laminate [45/0/-45/90 $]_{2 s}$. For both types of laminates, each unidirectional ply is modeled as a homogeneous orthotropic solid whose anisotropic elastic constants are identified above. The fiber direction, however, changes continuously along the curvature of the structure. The present analysis assumes that the plies are directly bonded to each other: the effects of interlayer resin-rich regions are not taken into account.

For the corner section of the laminate structure, the local xyz coordinate system is taken as shown in Fig. 1 (c) with the $x$ axis in the thickness direction and the $y$ axis along the curvature of the corner. With respect to this local system, the stiffness components are given by Eq. (2). For the unidirectional laminate $[0]_{16}$ the fiber direction is everywhere oriented along the corner, i.e., $\theta=0$, and for the quasi-isotropic laminate $[45 / 0 /-45 / 90]_{2 S}$ the angle $\theta$ takes either $\pi / 4,0,-\pi / 4$ or $\pi / 2$ in each ply. The stiffness components $C_{i j k l}^{X Y Z}$ at each material point with respect to the global $X Y Z$ system can be obtained from the stiffness components $C_{i j k l}^{x y z}$ with respect to the local xyz system using the transformation rule, i.e.,

$$
C_{i j k l}^{X Y Z}=R_{i m} R_{j n} R_{k s} R_{l t} C_{m n s t}^{x y z},
$$


(a) Corner model

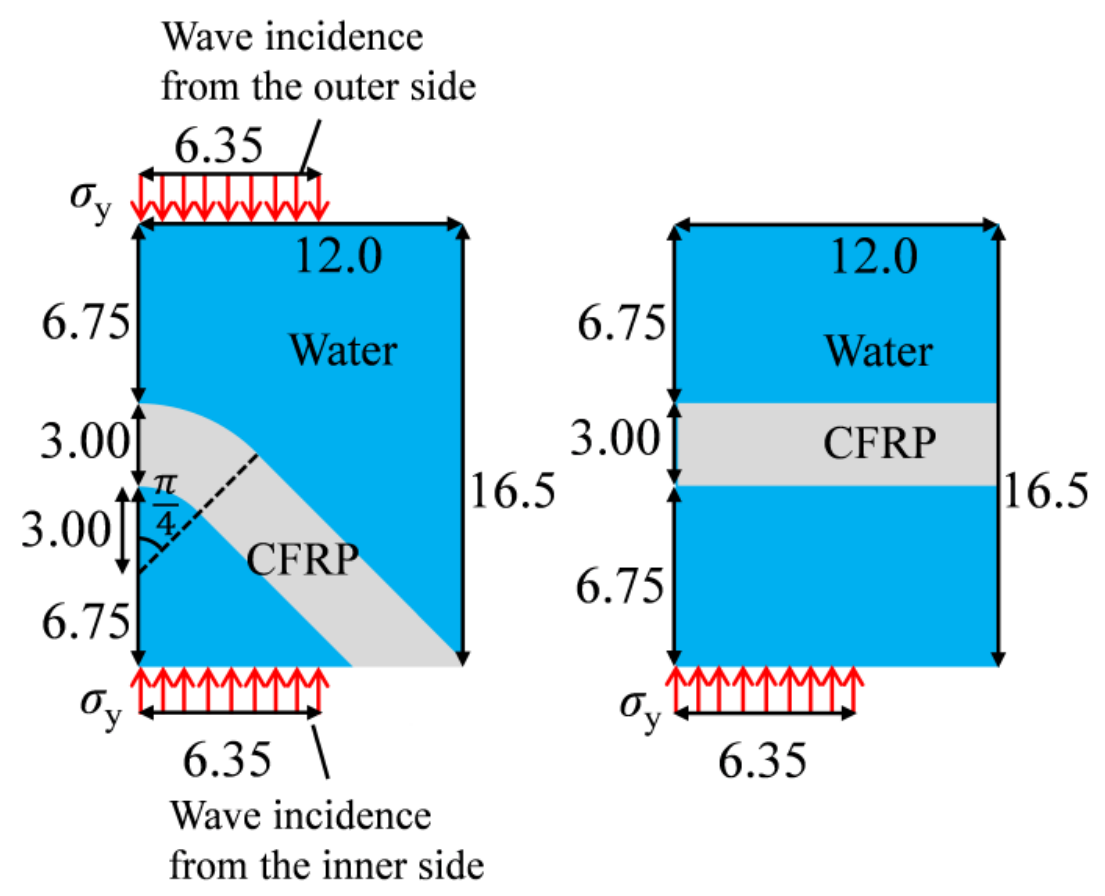

Fig. 2 Finite element model for (a) a corner section and (b) a plane laminate.

where $R_{i m}$ are the direction cosines given in the matrix form by

$$
[R]=\left[\begin{array}{ccc}
\cos \alpha & \sin \alpha & 0 \\
-\sin \alpha & \cos \alpha & 0 \\
0 & 0 & 1
\end{array}\right]
$$

as a function of the angular variable $\alpha$ giving the position along the corner as shown in Fig. 1 (c), where $\pi / 4<\alpha<3 \pi / 4$.

\subsection{Wave propagation analysis}

A commercial finite element analysis code MSC Marc (MSC Software Corporation) is used to simulate the ultrasonic wave propagation in the corner section of CFRP structure immersed in water. The wave propagation is analyzed as a two-dimensional plane-strain problem in the $X Y$ plane. Accounting for the symmetry of the structure and the wave field of interest, the right half of the structure shown in Fig. 2 (a) is modeled with 329,307 elements including four- and three-node isoparametric elements. Local elastic properties of CFRP structure are modeled in the manner described in the previous section. The water is modeled as an elastic solid with mass density 998 $\mathrm{kg} / \mathrm{m}^{3}$, bulk modulus $2.19 \mathrm{GPa}$ and null shear modulus.

In order to model the wave incidence from both inner and outer sides of the corner, the normal stress distribution is prescribed on a part of the top or the bottom boundary of the computational 
(a) Unidirectional
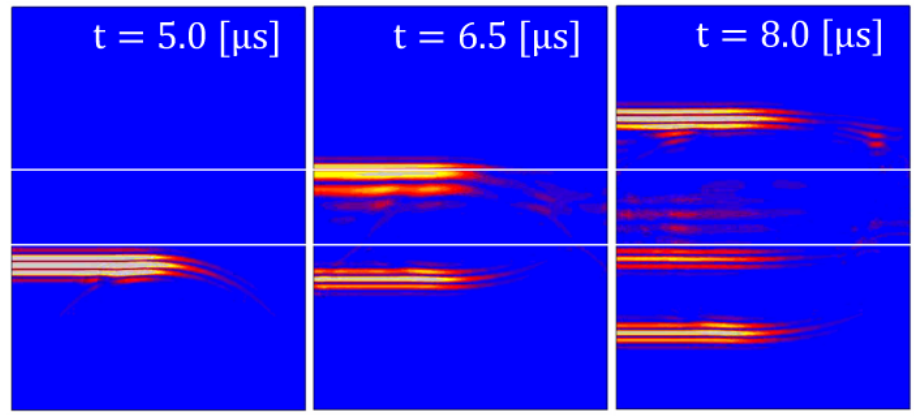

(b) Quasi-isotropic

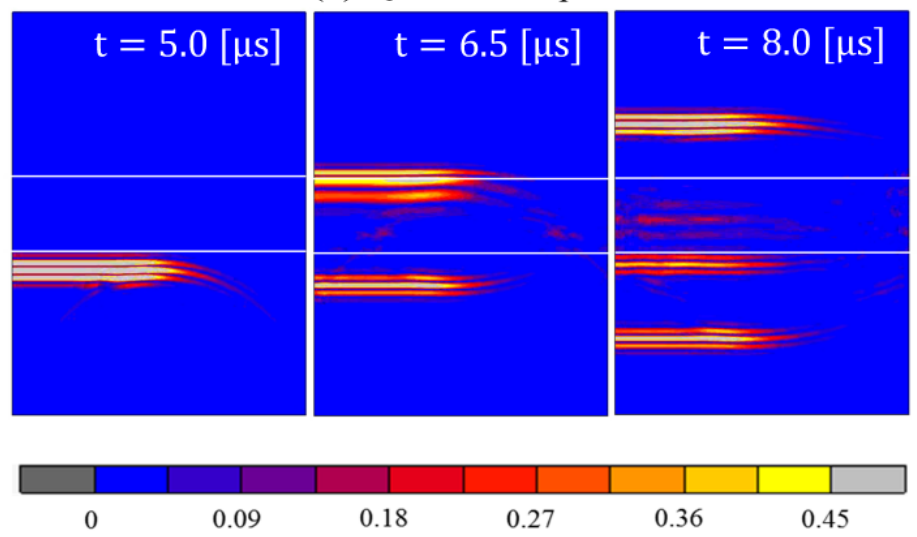

Fig. 3 Snapshots of wave propagation for the plane laminate model.

model. This part with the prescribed stress is referred to as the excitation boundary hereafter, and its half-width is $6.35 \mathrm{~mm}$ as shown in Fig. 2 (a). The wave excitation by a non-focusing flat transducer element of the center frequency of $2.25 \mathrm{MHz}$ is simulated by assuming

$$
\sigma_{y}(t)=\sigma_{0} \exp \left\{-\frac{\left(t-t_{0}\right)^{2}}{\tau_{0}^{2}}\right\} \cos \left\{2 \pi f_{0}\left(t-t_{0}\right)\right\},
$$

where $f_{0}=2.25$ (MHz), $t_{0}=1$ ( $\left.\mu \mathrm{s}\right)$ and $\tau_{0}=0.32$ ( $\left.\mu \mathrm{s}\right)$. In order to simulate the acquisition of the reflected signal by the same transducer element, the subsequent time history of the normal particle velocity is averaged over the excitation boundary. The particle velocity accompanying the prescribed stress waveform of Eq. (6) is also averaged over the excitation boundary, and its peak value is used as the amplitude of the incident wave. In the results shown below, the reflection waveforms are normalized by this incident wave amplitude. The amplitude parameter $\sigma_{0}$ in Eq. (6) can then be set arbitrarily as it merely plays a magnification factor due to the linearity of the analysis. The time integration is carried out in an implicit manner using the Newmark- $\beta$ method with the time step of $10 \mathrm{~ns}$.

For comparison, the wave propagation is also analyzed in a similar manner for a plane laminate structure of the same thickness as modeled in Fig. 2 (b), assuming the unidirectional laminate $[0]_{16}$ 
(a) Plane model

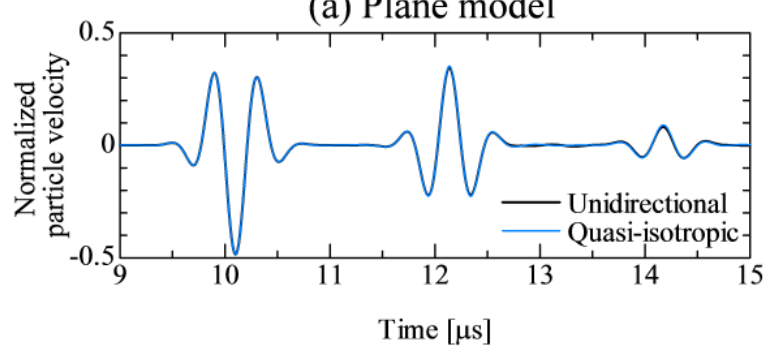

(b) Corner model (inner side)

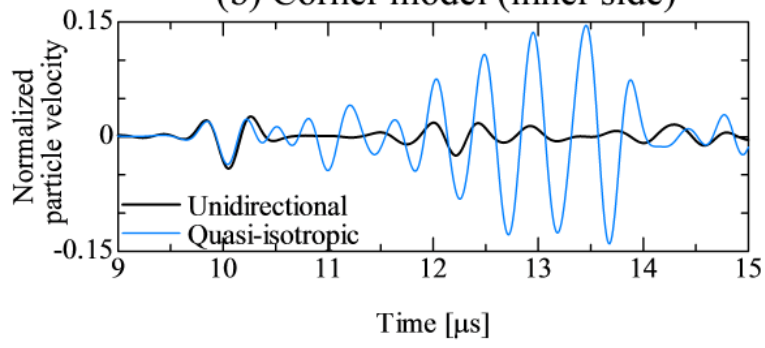

(c) Corner model (outer side)

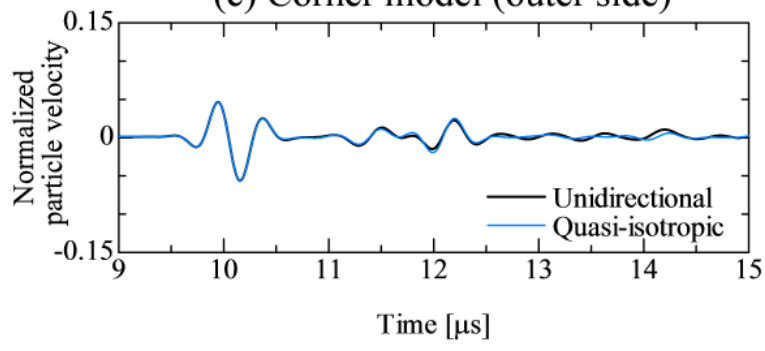

Fig. 4 Computed reflection waveforms for unidirectional and quasi-isotropic laminates, (a) plane laminate, (b) corner section for the wave incidence from the inner side, and (c) corner section for the wave incidence from the outer side.

and the quasi-isotropic laminate $[45 / 0 /-45 / 90]_{2 s}$. Since the stacking sequence is symmetric with respect to the mid-plane for both types of laminates, only the wave incidence from the bottom boundary is analyzed. For this plane laminate model, the distance between the excitation boundary and the surface of the laminate is the same $(6.75 \mathrm{~mm})$ as the corresponding distances for the corner model along the beam axis of the incident ultrasound. For the two models in Fig. 2, the left edge is treated as a symmetric boundary, and the other edges are assumed traction-free except that non-zero normal stress is prescribed as a function of time as given in Eq. (6) on the excitation boundary. The wave reflections from these artificial edges give no significant influence on the signal waveforms discussed below.

\section{Numerical results}

\subsection{Plane laminate}

Numerical results are first presented for the wave incidence in the normal direction to the plane laminate. In Fig. 3 (a) and (b), the distributions of the particle velocity magnitude at different elapsed times are shown for the plane unidirectional laminate and the plane quasi-isotropic laminate, respectively. The magnitude of the particle velocity is normalized by the incident wave amplitude as 
(a) Unidirectional
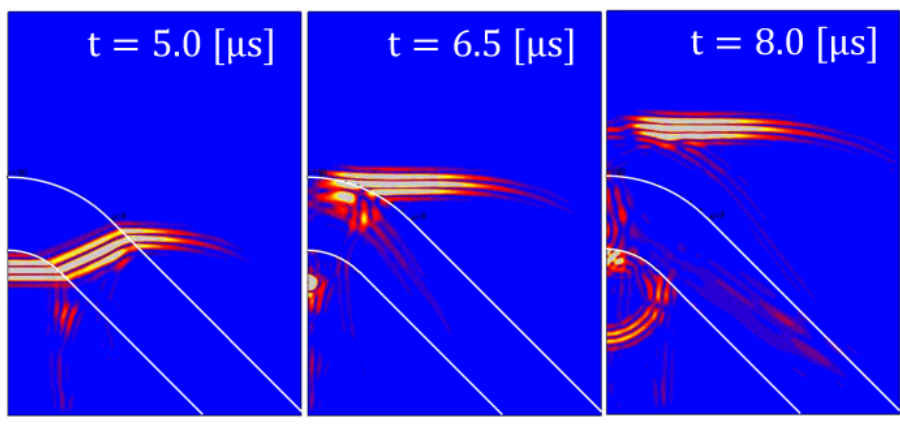

(b) Quasi-isotropic
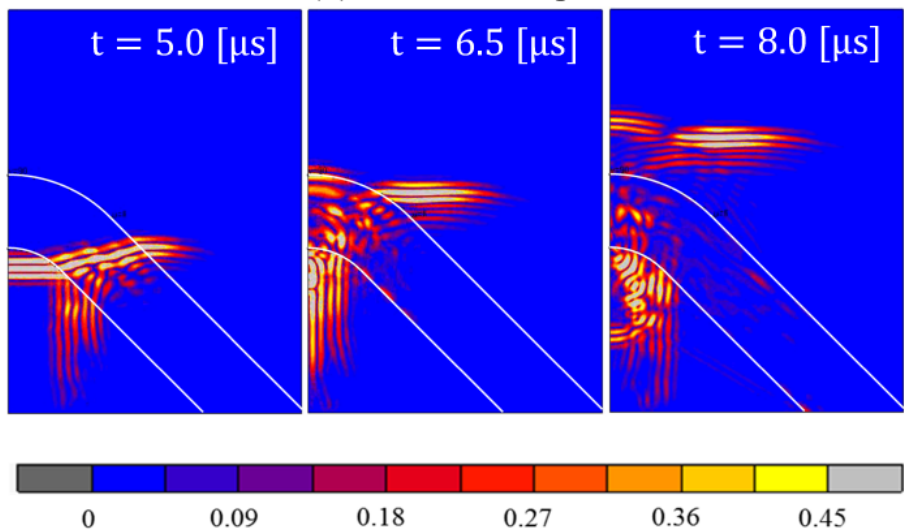

Fig. 5 Snapshots of wave propagation for the corner model when the wave is incident from the inner side.

explained in the previous section. In Fig. 3, the emitted ultrasonic wave appears to propagate in water and in the laminate essentially as a plane wave. The reflection waveforms for the two types of laminates are shown in Fig. 4 (a), where the surface echo (at about $10 \mu \mathrm{s}$ ), the first back-wall echo (at about $12 \mu \mathrm{s}$ ) and the second back-wall echo (at about $14 \mu \mathrm{s}$ ) are clearly recognized for both laminates. It is also found in Fig. 4 (a) that the signals for both laminates are almost identical. This is a natural consequence since all plies have the same longitudinal wave velocity and acoustic impedance in the thickness direction.

\subsection{Corner section for the wave incidence from the inner side}

In Fig. 5 (a) and (b), the snapshots of the particle velocity magnitude at different elapsed times are shown in the case where the wave is incident from the inner side of the corner section. As shown in Fig. 5, the wave first encounters the structure at its plane surface and the refracted wave propagates towards the corner. The wave reflected from the corner tends to focus at a point in water and spreads again before it arrives back at the excitation boundary.

The reflection waveforms are shown in Fig. 4 (b) for both types of laminates. Due to the wave propagation behavior seen in the snapshots of Fig. 5, the waveforms reflect the material properties of a wide area of the structure in a more complex manner as compared to the case of plane laminates. 
(a) Unidirectional

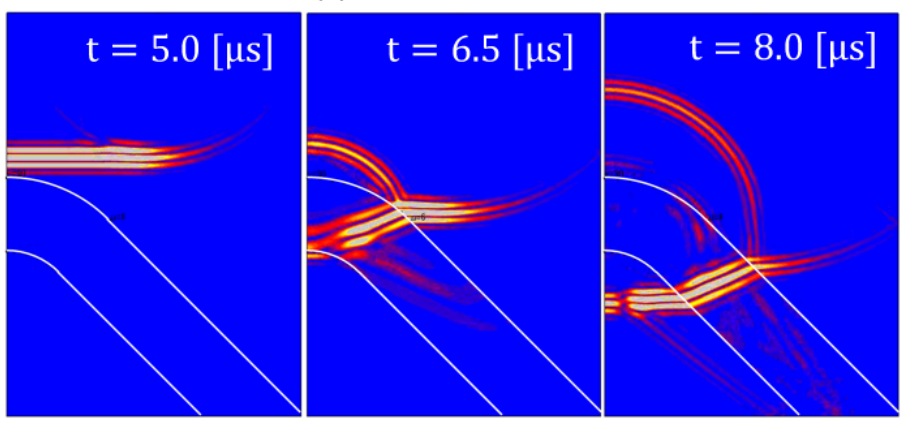

(b) Quasi-isotropic
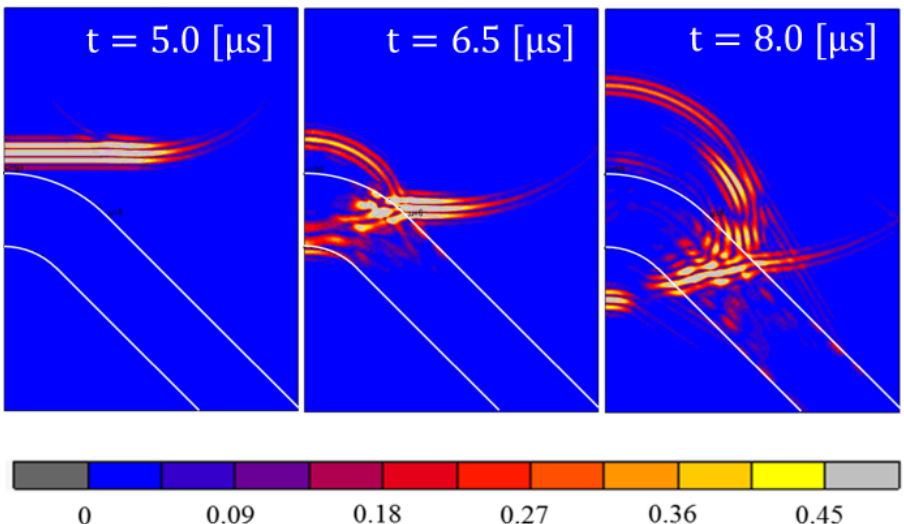

Fig. 6 Snapshots of wave propagation for the corner model when the wave is incident from the outer side.

For the unidirectional laminate shown in Fig. 4 (b), the surface echo can be seen at about $10 \mu \mathrm{s}$, but at about $12 \mu \mathrm{s}$, a direct back-wall echo along the beam axis and an echo which has refracted and traveled along the corner are both present. For the quasi-isotropic laminate, the waveform is even more complex as it contains reflection from interlayer interfaces, and the back-wall echo cannot be distinguished clearly. These results show that the stacking sequence of the laminate has a remarkable effect on the reflection waveform when the corner section is probed from its inner side. Furthermore, the reflection signals are significantly weak as compared to those of the plane laminates.

\subsection{Corner section for the wave incidence from the outer side}

The snapshots of wave propagation in the case of wave incidence from the outer side of the corner section are shown in Fig. 6 (a) and (b). In this case, the wave is reflected in a wide angle at the surface and the back-wall of the corner section. The refracted component of the wave propagates along the laminate structure and does not travel back to the excitation boundary. Therefore, the reflection waveform mainly bears information of material properties of a relatively narrow area along the ultrasonic beam axis.

The corresponding reflection waveforms are shown in Fig. 4 (c) for the unidirectional and 


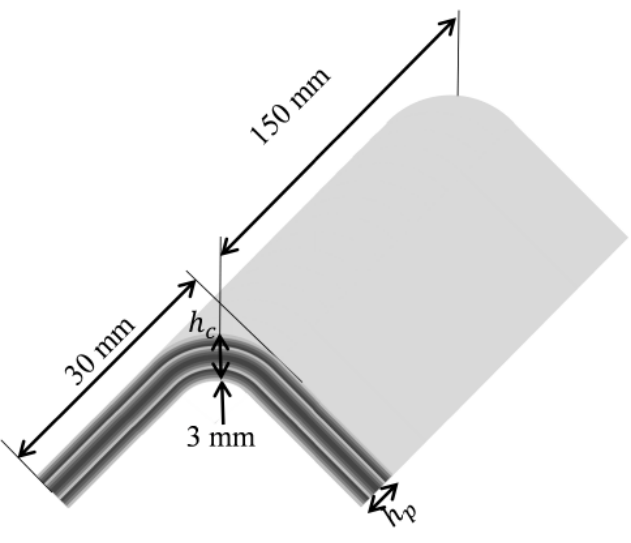

Fig. 7 Fabricated specimen with corner section.
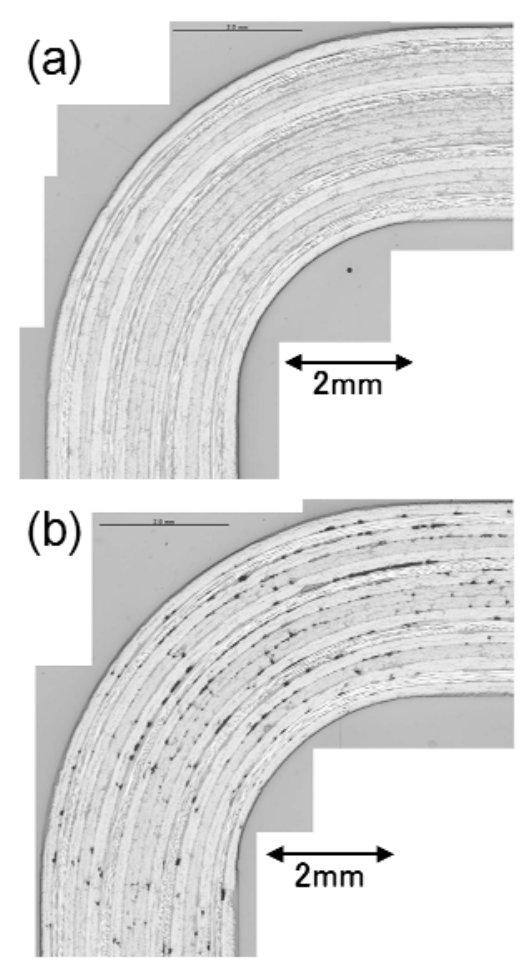

Fig. 8 Cross-sections of the corner section of quasi-isotropic laminate, (a) normal specimen, (b) specimen with porosity $1.6 \%$.

quasi-isotropic laminates. At a glance, the waveforms resemble those for the plane laminates shown in Fig. 4 (a) with the surface and back-wall echoes being clearly distinguishable. The echoes in Fig. 4 (c) are, however, significantly weak as compared to those in Fig. 4 (a). This is due to the difference in the reflected wave fields in the two cases. Namely, the corner section produces a cylindrical wave front (Fig. 6), while the plane laminate makes a nearly plane wave front (Fig. 3). Consequently, less energy will propagate back to the excitation boundary from the corner section. Furthermore, the phase of the reflected wave from the corner section is not constant along the excitation boundary, which results in a smaller signal when the particle velocity is spatially averaged.

As seen in Fig. 4 (c), the reflection waveforms are almost independent of the stacking sequence. This is a feature also seen for the plane laminates in Fig. 4 (a). It is mainly due to the fact that the echoes from interlayer interfaces in the quasi-isotropic laminate rarely propagate back to the incidence direction due to the curvature of the structure.

\section{Experiments}

CFRP laminate specimens having a right-angle corner section were manufactured using the same prepregs as described in Section 2.1. The corner section of each specimen has nominal inner radius 3 $\mathrm{mm}$ and thickness $3 \mathrm{~mm}$. The measured dimensions of the specimens are shown in Fig. 7. Specimens were fabricated with two kinds of stacking sequence as modeled above, i.e. the unidirectional 
(a) Plane section

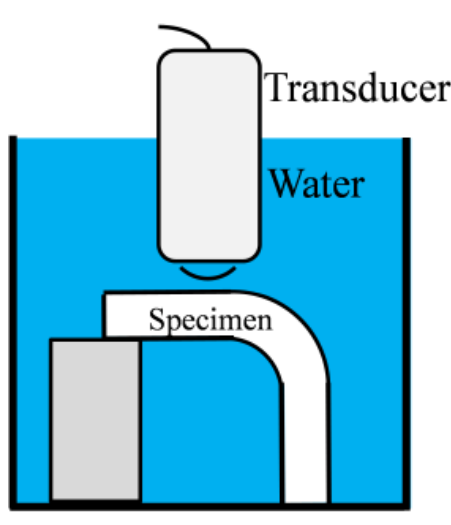

(b) Corner section

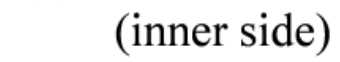

(c) Corner section (outer side)

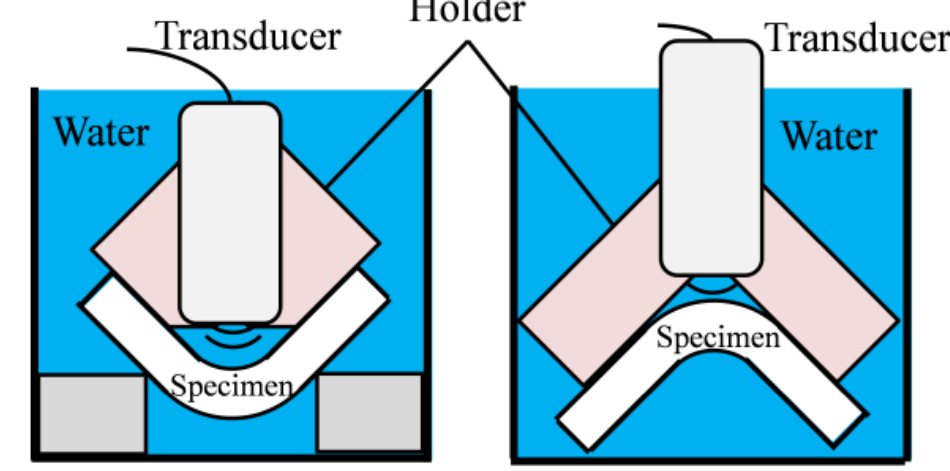

Fig. 9 Schematic illustration of reflection measurements.

laminate $[0]_{16}$ and the quasi-isotropic laminate $[45 / 0 /-45 / 90]_{2 S}$. These specimens are nominally porosity-free, and referred to as normal specimens in the following discussions.

In addition to the above normal specimens, similar specimens having different levels of porosity were also fabricated by reducing the applied pressure intentionally in the curing process. The porosity contents of these specimens were estimated as an area ratio of pores on the optical micrograph of their side surfaces. The measured thicknesses and the evaluated porosity contents at their corner section and at their plane section are shown in Table 1. Figure 8 show optical micrographs of the side surfaces of (a) the normal specimen and (b) the specimen with porosity content $1.6 \%$ of quasi-isotropic laminates.

Ultrasonic reflection measurements were carried out for the plane section as well as for the corner section of the specimens described above. Each specimen was immersed in water as schematically shown in Fig. 9 and supported by aluminum blocks when it was necessary to face the plane surface or the inner side of the corner to the emitting/receiving ultrasonic probe, which was a non-focusing type described in Section 2.1 with nominal center frequency of $2.25 \mathrm{MHz}$. The probe was set in a special holder to keep the incidence direction perpendicular to the specimen surfaces and the distance at $6.75 \mathrm{~mm}$ nominally. The probe was excited by a spike signal to emit a broadband longitudinal wave to the specimen, and the reflected wave was recorded by the same probe and stored in a personal computer via a digital oscilloscope after averaged for 128 waveforms.

\section{Experimental results and discussion}

\subsection{Reflection waveforms for normal specimens}

The recorded reflection waveforms for the normal specimens are shown in Fig. 10 (a) for the case when the wave is incident on the plane section in the perpendicular direction, and in Fig. 10 (b) and (c) when the wave is incident on the corner section from its inner and outer sides, respectively. In Fig. 10 (a)-(c), the measured waveforms are shown for the unidirectional laminate and the quasi-isotropic laminate. 
(a) Plane section

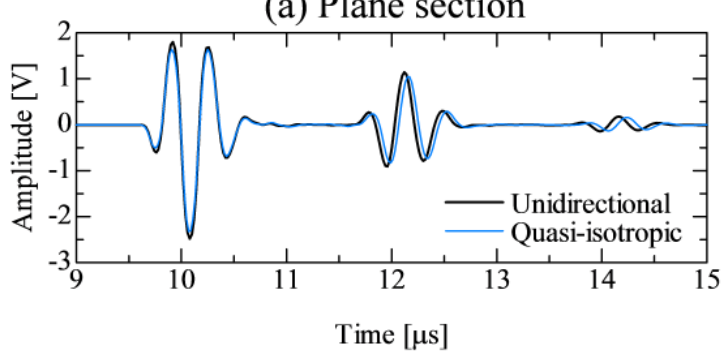

(b) Corner section (inner side)

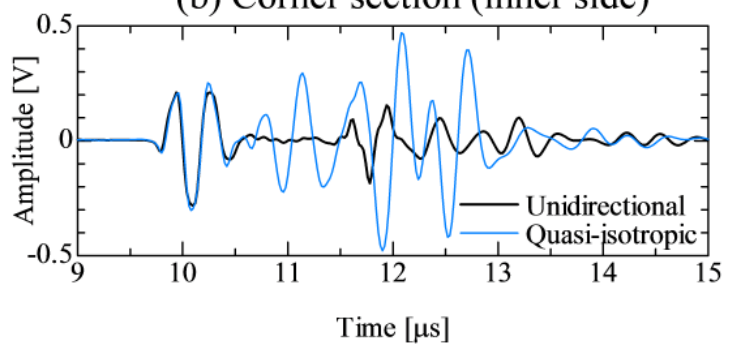

(c) Corner section (outer side)

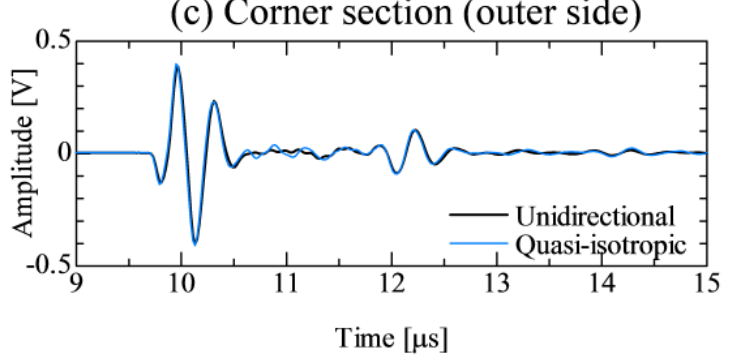

Fig. 10 Measured reflection waveforms for unidirectional and quasi-isotropic laminates, (a) plane section, (b) corner section for the wave incidence from the inner side, and (c) corner section for the wave incidence from the outer side.

In the reflection waveforms for the plane section (Fig. 10 (a)), the surface, the first back-wall and the second back-wall echoes are clearly seen at about 10, 12 and $14 \mu$ s, respectively. Two waveforms in Fig. 10 (a) are almost identical in spite of the difference in the stacking sequence, although there is a slight delay of the back-wall echoes in the waveform for the quasi-isotropic structure. A qualitatively similar trend can be seen in the reflection waveforms for the corner section when the wave is incident from the outer side (Fig. 10 (c)), which have clearly distinguishable surface and first back-wall echoes independent of the stacking sequence. The reflection signals in Fig. 10 (c) are significantly weak compared to those in Fig. 10 (a). In contrast, the reflection waveforms of the corner section for the wave incidence from the inner side (Fig. 10 (b)) show remarkable difference between the two laminate structures. In Fig. 10 (b), the waveform for the unidirectional laminate has a clear surface echo but the first and second back-wall echoes are not clearly separated. The waveform for the quasi-isotropic laminate shows a complicated signal following the surface echo, which makes the back-wall echoes hardly recognizable.

Qualitatively, the above features seen in the measured reflection waveforms for the plane and the 
(a) Unidirectional

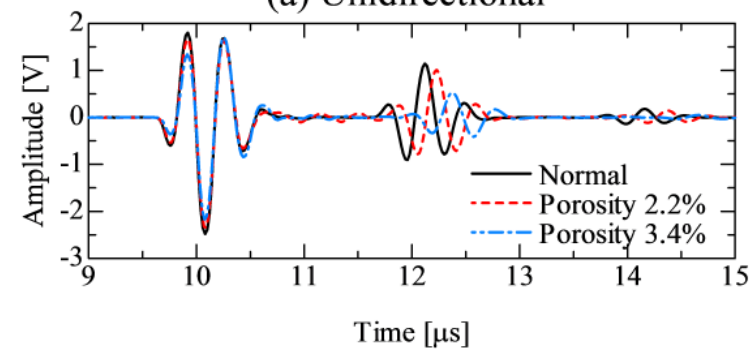

(b) Quasi-isotropic

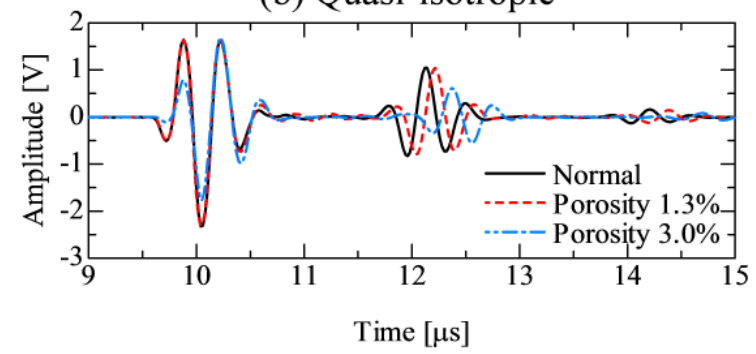

Fig. 11 Measured reflection waveforms for the plane section of (a) unidirectional and (b) quasi-isotropic laminates, for different porosity contents.

(a) Unidirectional

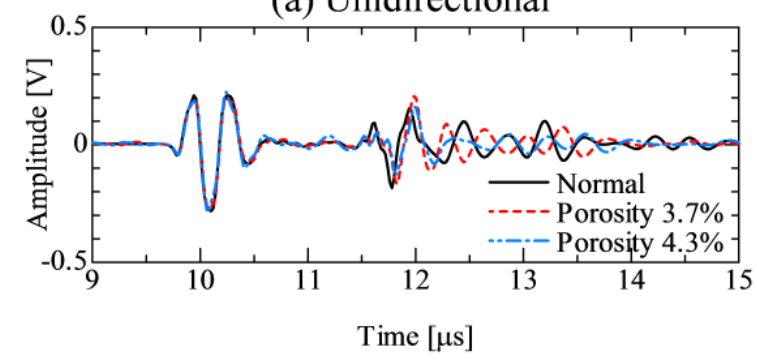

(b) Quasi-isotropic

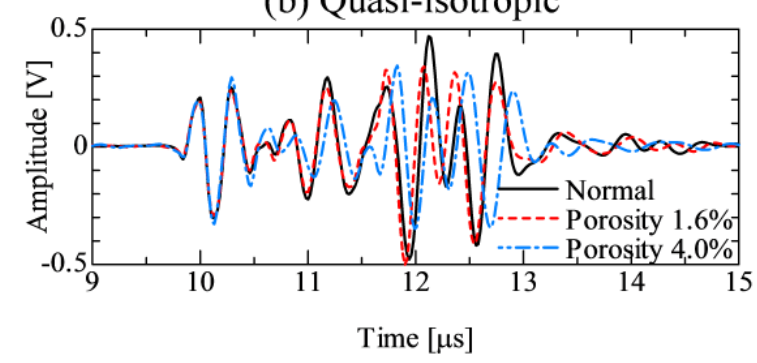

Fig. 12 Measured reflection waveforms for the corner section of (a) unidirectional and (b) quasi-isotropic laminates when the wave is incident from the inner side, for different porosity contents.

corner sections are well reproduced by the numerical simulations described in Section 3. Due to the two-dimensional nature of the simulations, the quantitative comparison between the analysis and experiments is beyond the scope of the present study. Furthermore, the experimental results contain the influence of ultrasonic wave attenuation in CFRP due to its viscoelastic properties [26] which are 
(a) Unidirectional

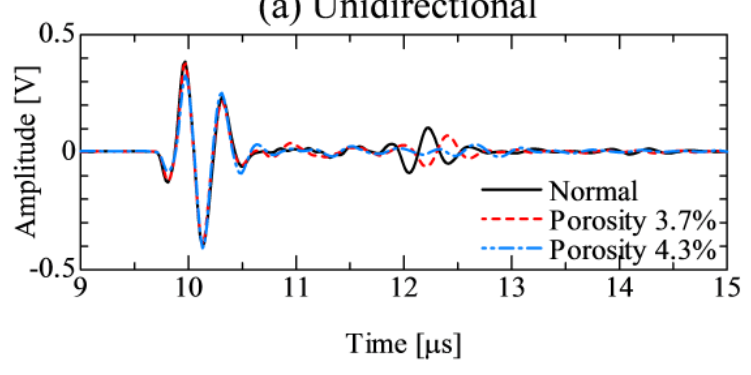

(b) Quasi-isotropic

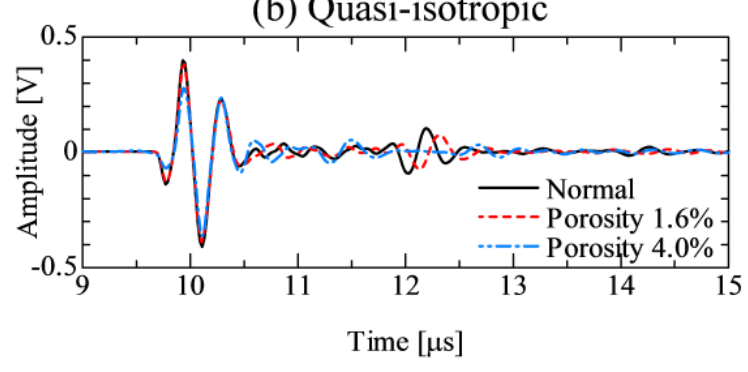

Fig. 13 Measured reflection waveforms for the corner section of (a) unidirectional and (b) quasi-isotropic laminates when the wave is incident from the outer side, for different porosity contents.

neglected in the numerical simulations. From the numerical simulations and experiments, however, it is shown that the corner section of CFRP laminate can be probed from the outer side to obtain clearly recognizable surface and back-wall echoes, whose arrival times and amplitudes are expected to reflect the local material characteristics. As an example, the effect of porosity on the reflection waveforms for the corner sections is discussed in the following section.

\subsection{Reflection waveforms for the specimens with porosity}

The recorded reflection waveforms for the plane sections with different porosity contents are shown in Fig. 11 (a) and (b) for the unidirectional and quasi-isotropic laminates, respectively. The waveforms for the normal specimens are also shown for comparison. In Fig. 11 (a) and (b), it is clearly seen that the first back-wall echoes are delayed and more attenuated with increasing porosity content. The corresponding waveforms for the corner sections are shown in Figs. 12 and 13 for the wave incidence from the inner and outer sides, respectively. For the wave incidence from the inner side (Fig. 12), it is not easy to observe a systematic effect of the porosity content on the reflection waveforms. In contrast, for the wave incidence from the outer side (Fig. 13), delay and amplitude reduction of the first back-wall echoes due to porosity can be found clearly.

The above results indicate that the porosity content of the corner sections can be probed in a similar manner to the plane sections when the ultrasound is incident from the outer side. In order to examine this further, the time difference of the surface and the first back-wall echoes was obtained for different locations of each specimen by the zero-crossing method to roughly estimate the wave velocity in the thickness direction. The resulting relation between the estimated wave velocity and the porosity content is shown in Fig. 14. For the plane sections, the wave velocity decreases as the 


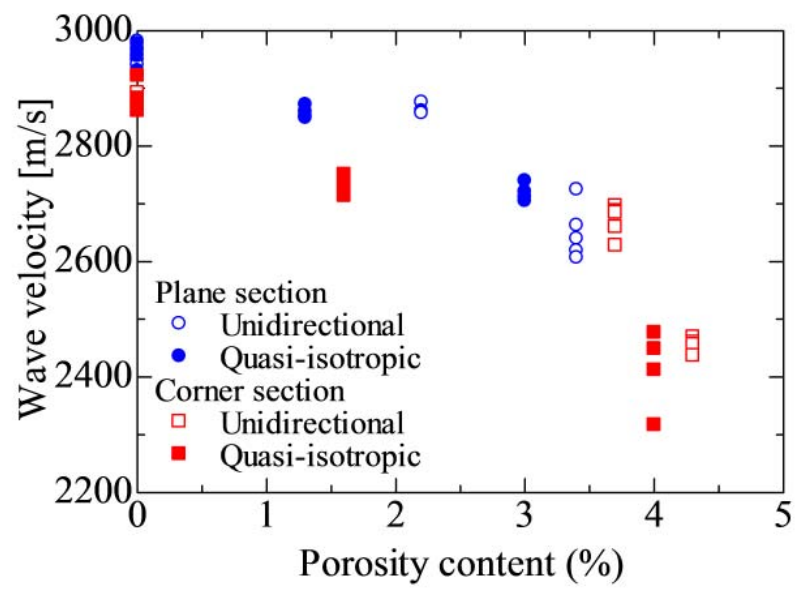

Fig. 14 Variation of the estimated wave velocity with porosity content.

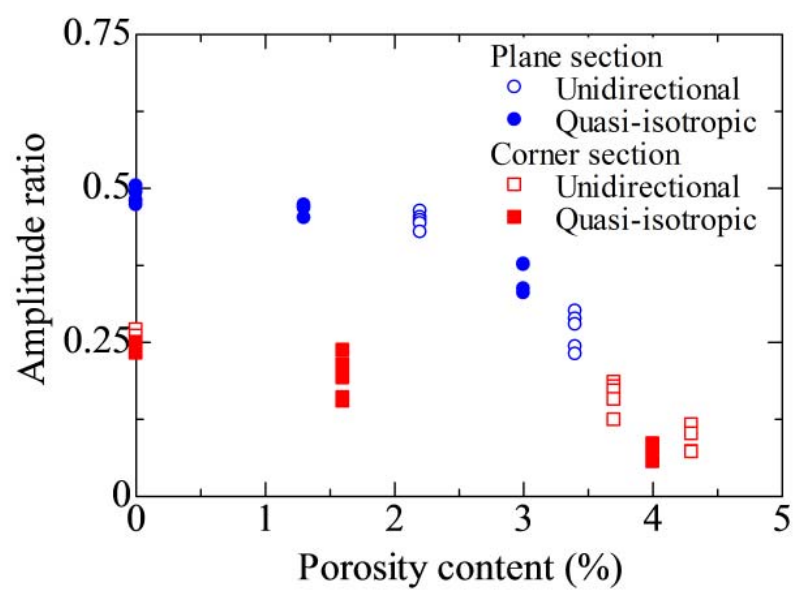

Fig. 15 Variation of the amplitude ratio of surface and first back-wall echoes with porosity content.

porosity content increases. The results for the corner sections show a similar trend to those for the plane sections, although the estimation of wave velocity for the corner sections is less accurate due to the curvature of the specimens and the cylindrical nature of reflected wave fronts.

The ratio of the peak-to-peak amplitude of the surface and the first back-wall echoes, referred to as the amplitude ratio, was calculated for each specimen and plotted in Fig. 15 against the porosity content. This ratio is used here as a measure of ultrasonic attenuation due to wave scattering loss by pores, although more quantitative evaluation of attenuation characteristics requires the compensation for the beam spreading loss due to the curvature of specimens and the interface transmission loss. For the plane sections, the amplitude ratio decreases as the porosity content increases, with a steeper slope beyond the porosity level of about 2 percent. For the corner sections, the amplitude ratio also appears to decrease but more gradually with the porosity content. It is noted that the amplitude ratios for the corner sections are substantially lower than those for the plane sections, likely due to the cylindrical nature of wave fronts as clarified in the numerical simulations. As a consequence, the 
evaluation of porosity content can be performed for the corner section using echo amplitudes, but it should be based on different calibration relations between the echo amplitude and the porosity content for different curvatures of the corner.

As an additional remark, the present numerical analysis and experiments indicate the difficulty of extracting information of material property of composite corner sections when the ultrasound is incident from the inner side of the curvature. It should be noted that the present study, being meant to be of a fundamental nature, assumed the use of a non-focusing probe. The use of focusing or array probes may solve this difficulty [16] and facilitate the testing from the inner side of the corner, which is often preferable in practical situations. The numerical simulations and experimental verifications for such situations remain as a topic for future investigations.

\section{Conclusion}

The two-dimensional finite element simulations have been presented for ultrasonic wave propagation behavior in a corner section of CFRP laminate structure. Numerical results have been demonstrated for the transient wave field and the reflection waveform when a broadband longitudinal wave from a non-focusing flat transducer is incident from the inner or the outer side of the corner. Numerical results have shown that when the corner section is probed from its outer side, the reflection waveform appears to be qualitatively similar to that for the plane laminate irrespective of the stacking sequence, and contains information of local material property. On the other hand, when probed from the inner side of the corner, the reflection waveform is significantly affected by the stacking sequence and is difficult to interpret. Reflection measurements have also been performed for CFRP corner specimens. Qualitative features of experimental reflection waveforms have been well reproduced by the finite element simulations. The experimental results have shown that the porosity content of the corner section can be estimated using the amplitude of the back-wall echoes for the wave incidence from the outer side, when an appropriate calibration relation based on the corner curvature is used.

\section{Acknowledgment}

The authors wish to thank Mr. Michio Ohno, Kawaju Gifu Engineering Co., for his contribution in manufacturing the specimens with controlled porosity levels.

\section{References}

[1] W. J. Cantwell and J. Morton, Detection of impact damage in CFRP laminates, Compos. Struct. 3 (1985), 241-257.

[2] R. D. Adams and P. Cawley, A review of defect types and nondestructive testing techniques for composites and bonded joints, NDT Int. 21 (1988), 208-222.

[3] C. Scarponi and G. Briotti, Ultrasonic detection of delaminations on composite materials, J. Reinf. Plast. Compos. 16 (1997), 768-790.

[4] C. Scarponi and G. Briotti, Ultrasonic technique for the evaluation of delaminations on CFRP, GFRP, KFRP composite materials, Composites B 31 (2000), 237-243.

[5] D. E. W. Stone and B. Clarke, Ultrasonic attenuation as a measure of void content in carbon fibre reinforced plastics, Non-Destructive Testing 8 (1975), 137-145. 
[6] J. M. Hale and J. N. Ashton, Ultrasonic attenuation in voided fibre-reinforced plastics, NDT Int. 21 (1988), 321-326.

[7] I. M. Daniel, S. C. Wooh and I. Komsky, Quantitative porosity characterization of composite materials by means of ultrasonic attenuation measurements, J. Nondestruct. Eval. 11 (1992), $1-8$.

[8] N. Guo and P. Cawley, The non-destructive assessment of porosity in composite repairs, Composites 25 (1994), 842-850.

[9] H. Jeong, Effects of voids on the mechanical strength and ultrasonic attenuation of laminated composites, J. Compos. Mater. 31 (1997), 276-292.

[10] E. A. Birt and R. A. Smith, A review of NDE methods for porosity measurement in fibre-reinforced polymer composites, Insight 46 (2004), 681-686.

[11] J.-W. Park, D.-J. Kim, K.-H. Im, S.-K. Park, D. K. Hsu, A. H. Kite, S.-K. Kim, K.S. Lee and I.-Y. Yang, Ultrasonic influence of porosity level on CFRP composite laminates using Rayleigh probe waves, Acta Mech. Solida Sinica 21 (2008), 298-307.

[12] L. Lin, X. Zhang, J. Chen, Y. Mu and X. Li, A novel random void model and its application in predicting void content of composites based on ultrasonic attenuation coefficient, Appl. Phys. A 103 (2011), 1153-1157.

[13] S. Deydier, N. Gengembre, P. Calmon, V. Mengeling and O. Pétillon, Ultrasonic field computation into multilayered composite materials using a homogenization method based on ray theory, AIP Conf. Proc. 760 (2005), 1057-1064.

[14] S. Journiac, N. Leymarie, N. Dominguez and C. Potel, Simulation of ultrasonic inspection of composite using bulk waves: application to curved components, J. Phys. Conf. Series 269 (2011), 012022.

[15] N. Dominguez, O. Grellou and S. Van-der-Veen, Simulation of ultrasonic NDT in composite radius, 10th European Conference on Non-Destructive Testing (Moscow, June 2010), 1-10-41.

[16] N. Xu and Z. Zhou, Numerical simulation and experiment for inspection of corner-shaped components using ultrasonic phased array, NDT \& E Int. 63 (2014), 28-34.

[17] Z. You, M. Lusk, R. Ludwig and W. Lord, Numerical simulation of ultrasonic wave propagation in anisotropic and attenuative solid materials, IEEE Trans. Ultras. Ferroelectr. Freq. Control 38 (1991), 436-445.

[18] D. Datta and N. N. Kishore, Features of ultrasonic wave propagation to identify defects in composite materials modelled by finite element method, NDT \& E Int. 29 (1996), 213-223.

[19] W. Ke, M. Castaings and C. Bacon, 3D finite element simulations of an air-coupled ultrasonic NDT system, NDT \& E Int. 42 (2009), 524-533.

[20] A. Castellano, P. Foti, A. Fraddosio, S. Marzano and M. D. Piccioni, Mechanical characterization of CFRP composites by ultrasonic immersion tests: experimental and numerical approaches, Composites B 66 (2014), 299-310.

[21] F. Moser, L. J. Jacobs and J. Qu, Modeling elastic wave propagation in waveguides with the finite element method, NDT \& E Int. 32 (1999), 225-234.

[22] T. Furukawa and I. Komura, Simulation and visualization of guided wave propagation by large-scale 3D FEM, E-J. Adv. Maintenance 3 (2011), 92-101.

[23] S. E. Burrows, B. Dutton and S. Dixon, Laser generation of Lamb waves for defect detection: 
experimental methods and finite element modeling, IEEE Trans. Ultrason. Ferroelec. Freq. Control 59 (2012), 82-89.

[24] R. E. Smith, Ultrasonic elastic constants of carbon fibers and their composites, J. Appl. Phys. 43 (1972), 2555-2561.

[25] R. D. Kriz and W. W. Stinchcomb, Elastic moduli of transversely isotropic graphite fibers and their composites, Exp. Mech. 19 (1979), 41-49.

[26] B. Hosten, M. Deschamps and B. R. Tittmann, Inhomogeneous wave generation and propagation in lossy anisotropic solids: Application to the characterization of viscoelastic composite materials, J. Acoust. Soc. Am. 82 (1987), 1763-1770.

[27] E. P. Papadakis, T. Patton, Y.-M. Tsai, D. O. Thompson and R. B. Thompson, The elastic moduli of a thick composite as measured by ultrasonic bulk wave phase velocity, J. Acoust. Soc. Am. 89 (1991), 2753-2757.

[28] S. K. Sahay, R. A. Kline and R. Mignogna, Phase and group velocity considerations for dynamic modulus measurement in anisotropic media, Ultrasonics 30 (1992), 373-382.

[29] S. I. Rokhlin, W. Wang, Double through-transmission bulk wave method for ultrasonic phase velocity measurement and determination of elastic constants of composite materials, J. Acoust. Soc. Am. 91 (1992), 3303-3312.

[30] R. A. Kline, Nondestructive Characterization of Composite Media, 1992, Technomic Pub., Pennsylvania. 
Table 1 Density, thicknesses and porosity contents of specimens.

\begin{tabular}{cccccc} 
Specimen & Density & \multicolumn{2}{c}{ Thickness $(\mathrm{mm})$} & \multicolumn{2}{c}{ Porosity content (\%) } \\
& $\left(\mathrm{g} / \mathrm{cm}^{3}\right)$ & Corner section & Plane section & Corner section & Plane section \\
\hline Unidirectional & 1.55 & 3.02 & 3.03 & None & None \\
& 1.53 & 3.09 & 3.07 & 3.7 & 2.2 \\
& 1.50 & 3.15 & 3.14 & 4.3 & 3.4 \\
Quasi-isotropic & 1.54 & 3.02 & 3.05 & & None \\
& 1.53 & 3.04 & 3.10 & 1.6 & None \\
& 1.51 & 3.16 & 3.15 & 4.0 & 1.3 \\
& & & & & 3.0
\end{tabular}


Figure legends

Fig. 1 A unidirectional CFRP layer, (a) when the fiber direction is along the $y$ axis and (b) when the fiber direction is rotated from the $y$ axis by angle $\theta$, and (c) a corner section of CFRP laminated structure.

Fig. 2 Finite element model for (a) a corner section and (b) a plane laminate.

Fig. 3 Snapshots of wave propagation for the plane laminate model.

Fig. 4 Computed reflection waveforms for unidirectional and quasi-isotropic laminates, (a) plane laminate, (b) corner section for the wave incidence from the inner side, and (c) corner section for the wave incidence from the outer side.

Fig. 5 Snapshots of wave propagation for the corner model when the wave is incident from the inner side.

Fig. 6 Snapshots of wave propagation for the corner model when the wave is incident from the outer side.

Fig. 7 Fabricated specimen with corner section.

Fig. 8 Cross-sections of the corner section of quasi-isotropic laminate, (a) normal specimen, (b) specimen with porosity $1.6 \%$.

Fig. 9 Schematic illustration of reflection measurements.

Fig. 10 Measured reflection waveforms for unidirectional and quasi-isotropic laminates, (a) plane section, (b) corner section for the wave incidence from the inner side, and (c) corner section for the wave incidence from the outer side.

Fig. 11 Measured reflection waveforms for the plane section of (a) unidirectional and (b) quasi-isotropic laminates, for different porosity contents.

Fig. 12 Measured reflection waveforms for the corner section of (a) unidirectional and (b) quasi-isotropic laminates when the wave is incident from the inner side, for different porosity contents.

Fig. 13 Measured reflection waveforms for the corner section of (a) unidirectional and (b) quasi-isotropic laminates when the wave is incident from the outer side, for different porosity contents.

Fig. 14 Variation of the estimated wave velocity with porosity content.

Fig. 15 Variation of the amplitude ratio of surface and first back-wall echoes with porosity content. 